\author{
International Journal of Innovative Research in \\ Electrical, Electronics, Instrumentation and Control Engineering
}

Vol. 7, Issue 3, March 2019

\title{
Smart Plant Watering Patrollers
}

\author{
Dr. Shanti Mahesh ${ }^{1}$, Shivam Pandey ${ }^{2}$, SriRam P T $^{3}$ \\ Head of Department, Dept. of IS \& E, Atria Institue of Technology, Banglore, India ${ }^{1}$ \\ Student, Atria Institue of Technology, Banglore, India ${ }^{2,3}$
}

\begin{abstract}
The project is design to build an obstacle avoidance robotic vehicle using ultrasonic sensors for its movement and a line following robotic vehicle using infrared sensor for obstacle detection and also a speed behavioural robotic vehicle using speed sensors to detect the speed of robot for dispensing the water to plant saplings accordingly. The Line follower robot is a mobile machine that can detect and follow the line drawn on the floor. Generally, the path is predefined and can be either visible like a black line on a white surface with a high contrasted color or it can be invisible like a magnetic filed. Definitely, this kind of Robot should sense the line with its Infrared Ray (IR) sensors that installed under the robot. After that, the data is transmitted to the processor by specific transition buses. Proposes robotic vehicle that has an intelligence built in it such that it directs itself whenever an obstacle comes in its path. This robotic vehicle is built, using a micro-controller of AT mega 8 family. An ultrasonic sensor is used to detect any obstacle ahead of it and sends a command to the micro controller. Depending on the input signal received, the microcontroller redirects the robot to move in an alternate direction by actuating the motors which are interfaced to it through a motor driver.
\end{abstract}

Keywords: Infrared Ray (IR), Artificial Intelligence (AI), Sensors, Robotic vehicle

\section{INTRODUCTION}

Obstacle avoidance is a primary requirement of any autonomous mobile robot. Obstacle avoidance Robot is design to allow robot to navigate in unknown environment by avoiding collisions.[1] Obstacle avoiding robot senses obstacles in the path, avoid it and resumes its running..the line follower robot is one of the self-operating mobile machines that follows a line drawn on the floor. The path can be a visible black line on a white surface (reverse). Actually, the line follower robots are a perennial favourite of the small robot builder but definitely the tricky part is to make the line follower fast and smooth in its response. The wheel speed sensor was initially used to replace the mechanical linkage from the road wheels to the seepdometer, eliminating cable breakage and simplifying the gauge construction (elimination all moving parts except for the needle/spring assembly). With the advent of automated driving aids, such as electronic ABS, the sensor also provided wheel speed data to the controllers to assist the operator in maintaining control of the vehicle. The vehicle Speed sensor is also used for the proper shifting up of gears for the vehicle maintenance. .The most common wheel speed sensor system consists of a ferromagnetic toothed reluctor ring (tone wheel) and a sensor (which can be passive or active).

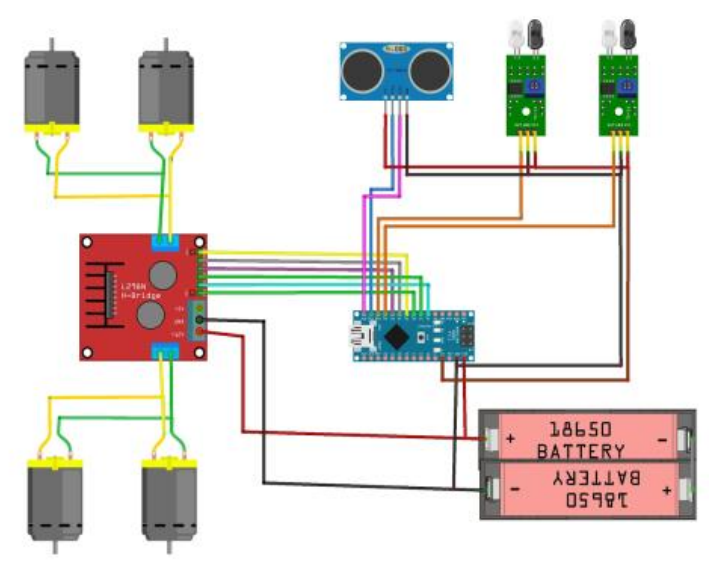

Figure (Design Of Speed Specific Robot) 


\section{International Journal of Innovative Research in Electrical, Electronics, Instrumentation and Control Engineering}

Vol. 7, Issue 3, March 2019

\section{EXISTING SYSTEM}

In simple robot, steering algorithm is used for robotic actions in which driver or a human being is controlling the robot using remote. Here driver is present, who can see the obstacle and navigate robot accordingly.

\section{PROPOSED SYSTEM}

The project proposes a autonomous robotic vehicle, In which no remote is used for controlling the robotic actions. It intelligently detects obstacles present on its path through the sensors and follows the path through sensors, as well as dispense the water to plant saplings by intergrated speed sensors. The detail information is given in the following subtopics which will help you to understand the whole system and its design.

\section{- BASIC DESIGN OF ROBOT}

This robot was built with an Arduino development board on which microcontroller is placed. Arduino board is connected with DC Motor through. Motor driver board(pin10, pin11, pin12, pin13)which provide power to the actuators. Actuators are used to move robot in Forward, Backward, Left and Right directions.

\begin{tabular}{|l|l|l|l|l|}
\hline Movement & Pin 10 & Pin 11 & Pin 12 & Pin 13 \\
\hline Forward & 1 & 0 & 0 & 1 \\
\hline Backward & 0 & 1 & 1 & 0 \\
\hline Left & 1 & 0 & 1 & 0 \\
\hline Right & 0 & 1 & 0 & 1 \\
\hline
\end{tabular}

The movement of robot will be stop whenever there is an obstacle is present on its path which can be detected by ultrasonic sensors. The correct path of the robot is detected by the Infrared sensor. The speed of the robot is sensed by the speed sensor and accordingly dispense the water in adequate amount.

\section{- SENSORS}

\section{- ULTRASONIC SENSOR}

In the design of robot, we are using ultrasonic sensors for obstacle detection and avoidance.[3]The ultrasonic sensors continuously emits the frequency signals, when obstacle is detected this signals are reflected back which then considered as input to the sensor.

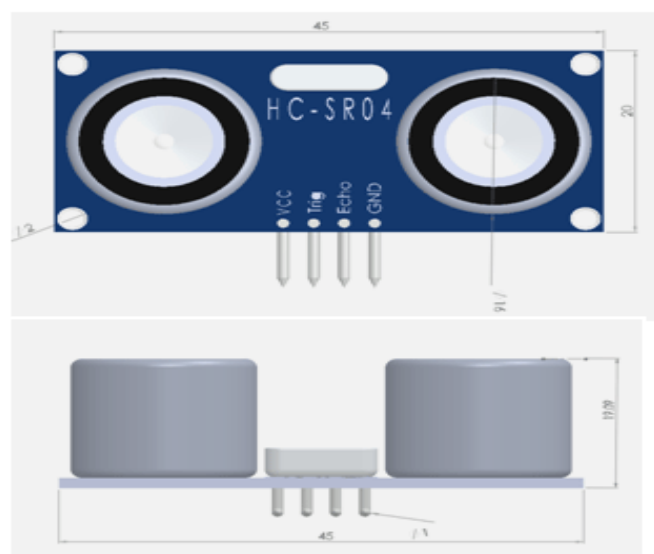

to calculate the distance using the above formulae, we should know the Speed and time. Since we are using the Ultrasonic wave we know the universal speed of US wave at room conditions which is $330 \mathrm{~m} / \mathrm{s}$.






\section{International Journal of Innovative Research in Electrical, Electronics, Instrumentation and Control Engineering}

Vol. 7, Issue 3, March 2019

\section{- INFRARED SENSOR}

An infrared sensor is an electronic device, that emits in order to sense some aspects of the surroundings. An IR sensor can measure the heat of an object as well as detects the motion. These types of sensors measures only infrared radiation, rather than emitting it that is called as a passive IR sensor

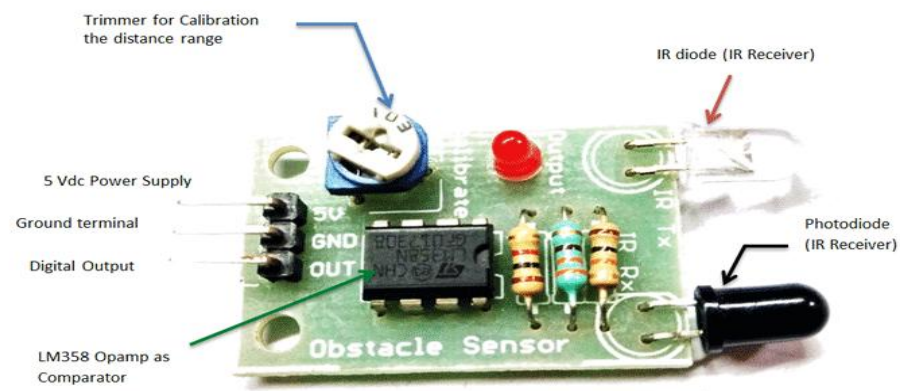

Usually in the infrared spectrum, all the objects radiate some form of thermal radiations. These types of radiations are invisible to our eyes, that can be detected by an infrared sensor.The emitter is simply an IR LED (Light Emitting Diode) and the detector is simply an IR photodiode which is sensitive to IR light of the same wavelength as that emitted by the IR LED



\section{- $\quad$ SPEED SENSOR} limit, etc.

The module can be used in association with a microcontroller for motor speed detection, pulse count, position
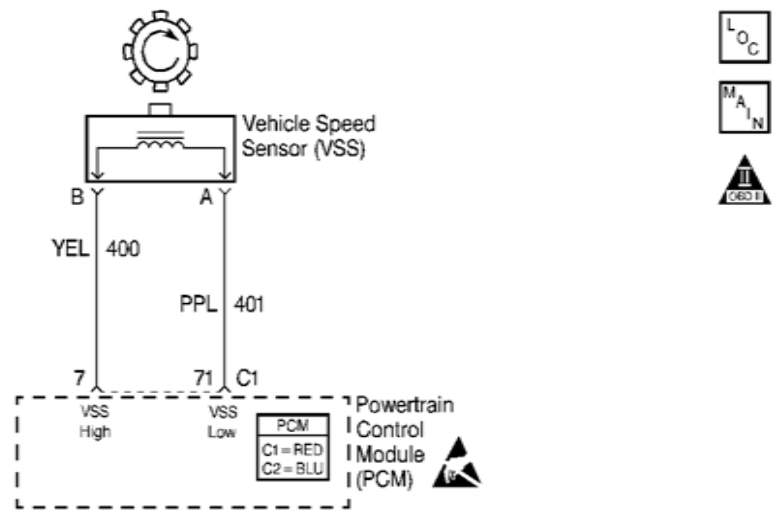

the circuity can be used to send calibrated pulses to a microcontroller-based tacho meter or similar circuit/device. The wiring of the hardware turned out to be deceptively simple. At the heart of the circuit is the OS25B10 transmissive optical sensor with phototransistor output (OC1). 


\section{International Journal of Innovative Research in Electrical, Electronics, Instrumentation and Control Engineering}

Vol. 7, Issue 3, March 2019

\section{- WORKING PRINCIPLE}

This is the main controlling unit of our model. It'll be responsible for the controlling of the process flow and sending signals to motor on different scenario. It is the smallest computer board which keeps looping to the code once the code is loaded.

IR transmits infrared lights. When infrared rays falls on white surface, it's reflected back and catched by photodiodes which generates some voltage changes. These Voltage change data are recognized by the conditional statements to detect the correct path.

\section{Smart Water Patrollers}

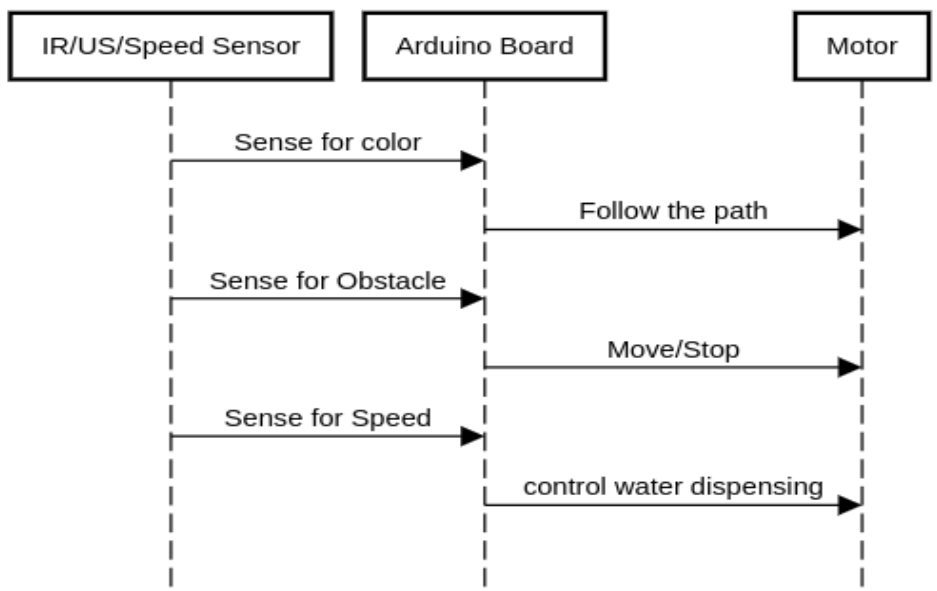

Ultrasonic sensors emit short, high-frequency sound pulses at regular intervals. If they strike an object, then they are reflected back as echo signals to the sensor and computes the distance. The reflected data received by arduino decide the distance and then sends the signal to motor .



\section{- IMPLEMENTATION}

Sensors are connected with the Arduino board using breadboard. Microcontroller is able to sense the environment through receiving input from sensors. It is also able to control its surrounding through controlling motors and other actuators.[5]

The Arduino programming language that is based on the processing are used to program the microcontroller found on the board. Due to its open-source environment, we can able to easily write and upload codes to the I/Oboard. Arduino environment is written in Javahence it can be run on Linux, Mac OSX and Windows platforms. 


\section{International Journal of Innovative Research in Electrical, Electronics, Instrumentation and Control Engineering}

Vol. 7, Issue 3, March 2019

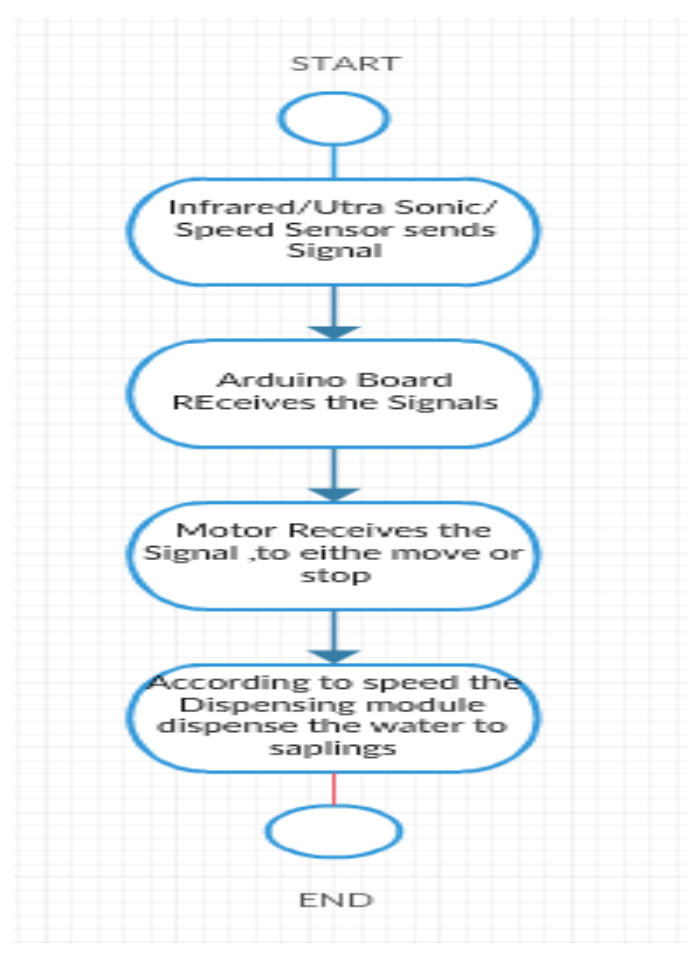

\section{CONCLUSION}

We build a robotic vehicle which moves in different directions like Forward, Backward, Left, and Right when input is given to it. The goal of our project is to create a autonomous robot which intelligently detects the obstacle in his path and navigate on a defined path and according to speed of robot dispense the water in adequate amount to plant saplings near roads.

\section{REFERENCES}

[1]. Obstacle avoidance robotic vehicle using ultrasonic sensors for obstacle detection: http://www.elprocus.com/obstacle-avoidance-robotic-vehicle/

[2]. How to make an obstacle avoiding robot: http://www.instructables.com/id/How-To-Make-an-Obstacle-Avoiding-Arduino-Robot/

[3]. IEEE paper for Obstacle avoidance robot using ultrasonic sensors:

http://www.personal.umich.edu/ ykoren/uploads/Obstacle_avoidance_w_ultrasonic_sensors_IEEE.pdf

[4]. Obstacle avoiding robot without microcontroller: http://www.roboticsbible.com/project-obstacle-avoiding-robot.html

[5]. How to build an obstacle avoiding robot: https://www.youtube.com/watch?v=JZ5JjvfY1Eg 